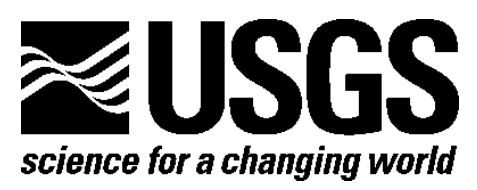

\title{
Mines and Mineral Processing Facilities in the Vicinity of the March 11, 2011, Earthquake in Northern Honshu, Japan
}

By W. David Menzie, Michael S. Baker, Donald I. Bleiwas, and Chin Kuo

Open-File Report 2011-1069

U.S. Department of the Interior

U.S. Geological Survey 


\section{U.S. Department of the Interior \\ KEN SALAZAR, Secretary}

\section{U.S. Geological Survey \\ Marcia K. McNutt, Director}

U.S. Geological Survey, Reston, Virginia: 2011

For more information on the USGS-the Federal source for science about the Earth, its natural and living resources, natural hazards, and the environment-visit http://www.usgs.gov or call 1-888-ASK-USGS

For an overview of USGS information products, including maps, imagery, and publications, visit $h$ ttp://www.usgs.gov/pubprod

To order this and other USGS information products, visit $h$ ttp://store.usgs.gov

Suggested citation:

Menzie, W.D., Baker, M.S., Bleiwas, D.I., and Kuo, Chin, 2011, Mines and mineral processing facilities in the vicinity of the March 11, 2011, earthquake in northern Honshu, Japan: U.S. Geological Survey Open-File Report 2011-1069, 7 p. (Available only at http://pubs.usgs.gov/of/2011/1069/.)

Any use of trade, product, or firm names is for descriptive purposes only and does not imply endorsement by the U.S. Government.

Although this report is in the public domain, permission must be secured from the individual copyright owners to reproduce any copyrighted material contained within this report. 


\section{Contents}

Mines and Mineral Processing Facilities in Northern Honshu, Japan ............................................................... 1

Requirements for Operation of the Mines and Mineral Processing Facilities ...................................................... 5

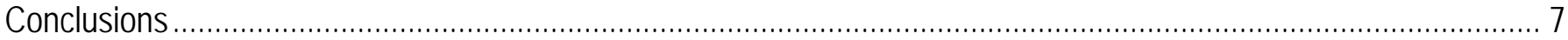

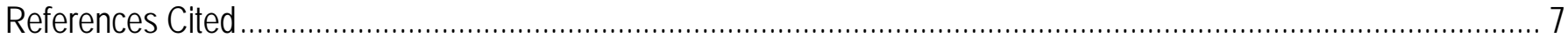

\section{Figures}

1. Map showing the location of mines and mineral facilities in Japan ......................................................... 2

2. Composite color image produced from satellite data captured at 10:07 UTC on March 9, 2011 .................. 6

3. Composite color image produced from satellite data captured at 9:30 UTC on March 12, 2011 ................... 7

\section{Table}

1. Mines and mineral processing facilities in northern Honshu, Japan ........................................................ 3 


\section{Mines and Mineral Processing Facilities in the Vicinity of the March 11, 2011, Earthquake in Northern Honshu, Japan}

By W. David Menzie, Michael Baker, Donald I. Bleiwas, and Chin Kuo

\section{Mines and Mineral Processing Facilities in Northern Honshu, Japan}

U.S. Geological Survey data indicate that the area affected by the March 11, 2011, magnitude 9.0 earthquake and associated tsunami is home to nine cement plants, eight iodine plants, four iron and steel plants, four limestone mines, three copper refineries, two gold refineries, two lead refineries, two zinc refineries, one titanium dioxide plant, and one titanium sponge processing facility (fig. 1, table 1). These facilities have the capacity to produce the following percentages of the world's nonfuel mineral production: 25 percent of iodine, 10 percent of titanium sponge (metal), 3 percent of refined zinc, 2.5 percent of refined copper, and 1.4 percent of steel. In addition, the nine cement plants contribute about one-third of Japan's cement annual production. The iodine is a byproduct from production of natural gas at the Miniami Kanto gas field, east of Tokyo in Chiba Prefecture. Japan is the world's second leading (after Chile) producer of iodine, which is processed in seven nearby facilities. 


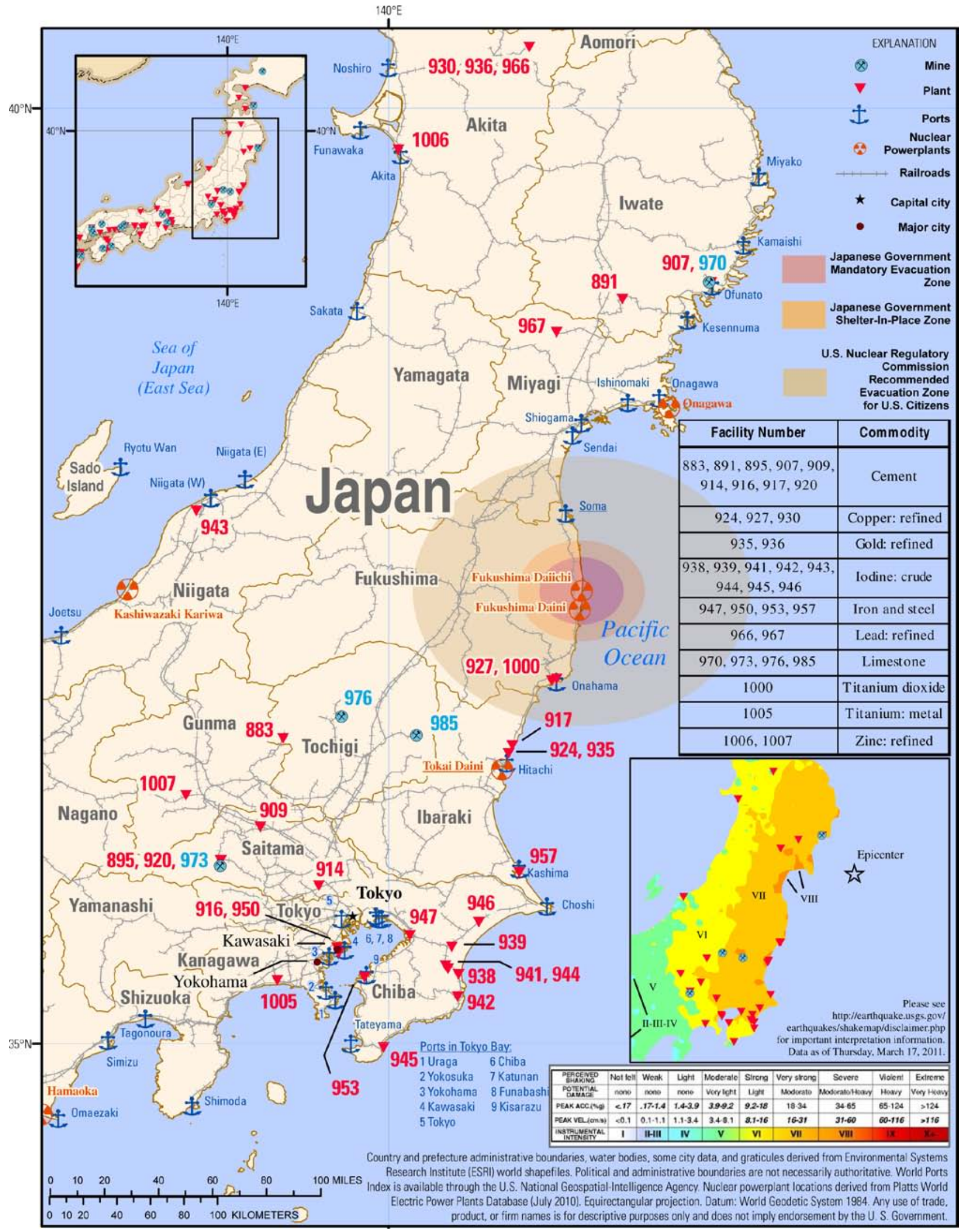

Figure 1. Map showing the location of mines and mineral facilities in Japan. Modified from Baker and others (2010). 
Table 1. Mines and mineral processing facilities in northern Honshu, Japan.

[NA, not available]

\begin{tabular}{|c|c|c|c|c|c|}
\hline $\begin{array}{l}\text { Facility no. } \\
\text { (fig. 1) }\end{array}$ & $\begin{array}{l}\text { Mineral } \\
\text { commodity }\end{array}$ & Location & $\begin{array}{l}\text { Facility } \\
\text { type }\end{array}$ & Operating company & Principal owner \\
\hline 883 & Cement & $\begin{array}{l}\text { Asio, Tochigi } \\
\text { Prefecture }\end{array}$ & Plant & $\begin{array}{l}\text { Sumitomo Osaka Cement Co. } \\
\text { Ltd. }\end{array}$ & NA. \\
\hline 891 & Cement & $\begin{array}{l}\text { Higashiyama, Iwate } \\
\text { Prefecture }\end{array}$ & Plant & Mitsubishi Materials Corp. & NA. \\
\hline 895 & Cement & $\begin{array}{l}\text { Yokoze, Saitama } \\
\text { Prefecture }\end{array}$ & Plant & Mitsubishi Materials Corp. & NA. \\
\hline 907 & Cement & $\begin{array}{l}\text { Ofunato, Iwate } \\
\text { Prefecture }\end{array}$ & Plant & Taiheiyo Cement Corp. & NA. \\
\hline 909 & Cement & $\begin{array}{l}\text { Kumagaya, Saitama } \\
\text { Prefecture }\end{array}$ & Plant & Taiheiyo Cement Corp. & NA. \\
\hline 914 & Cement & $\begin{array}{l}\text { Saitama, Saitama } \\
\text { Prefecture }\end{array}$ & Plant & Taiheiyo Cement Corp. & NA. \\
\hline 916 & Cement & $\begin{array}{l}\text { Kawasaki, Kanagawa } \\
\text { Prefecture }\end{array}$ & Plant & Daiichi Cement Co. Ltd. & NA. \\
\hline 917 & Cement & $\begin{array}{l}\text { Hitachi, Ibaraki } \\
\text { Prefecture }\end{array}$ & Plant & Hitachi Cement Co. Ltd. & NA. \\
\hline 920 & Cement & $\begin{array}{l}\text { Chichibu, Saitama } \\
\text { Prefecture }\end{array}$ & Plant & Taiheiyo Cement Corp. & NA. \\
\hline 924 & $\begin{array}{l}\text { Copper, } \\
\text { refined }\end{array}$ & $\begin{array}{l}\text { Hitachi, Ibaraki } \\
\text { Prefecture }\end{array}$ & Plant & Pan Pacific Copper Co. Ltd. & $\begin{array}{l}\text { Nippon Mining and Metals Co. } \\
\text { Ltd., 60\%. }\end{array}$ \\
\hline 927 & $\begin{array}{l}\text { Copper, } \\
\text { refined }\end{array}$ & $\begin{array}{l}\text { Onahama, Fukushima } \\
\text { Prefecture }\end{array}$ & Plant & $\begin{array}{l}\text { Onahama Smelting and Refining } \\
\text { Co. Ltd. }\end{array}$ & $\begin{array}{l}\text { Mitsubishi Materials Corp., } \\
\text { 49.29\%. }\end{array}$ \\
\hline 930 & $\begin{array}{l}\text { Copper, } \\
\text { refined }\end{array}$ & $\begin{array}{l}\text { Kosaka, Akita } \\
\text { Prefecture }\end{array}$ & Plant & $\begin{array}{l}\text { Kosaka Smelting and Refining } \\
\text { Co. Ltd. }\end{array}$ & $\begin{array}{l}\text { Dowa Metals and Mining Co. } \\
\text { Ltd., } 100 \% \text {. }\end{array}$ \\
\hline 935 & Gold, refined & $\begin{array}{l}\text { Hitachi, Ibaraki } \\
\text { Prefecture }\end{array}$ & Plant & Pan Pacific Copper Co. Ltd. & $\begin{array}{l}\text { Nippon Mining and Metals Co. } \\
\text { Ltd. 60\%. }\end{array}$ \\
\hline 936 & Gold, refined & $\begin{array}{l}\text { Kosaka, Akita } \\
\text { Prefecture }\end{array}$ & Plant & $\begin{array}{l}\text { Kosaka Smelting and Refining } \\
\text { Co. Ltd. }\end{array}$ & $\begin{array}{l}\text { Dowa Metals and Mining Co. } \\
\text { Ltd., } 100 \% .\end{array}$ \\
\hline 938 & Iodine, crude & $\begin{array}{l}\text { Ichinomiya, Chiba } \\
\text { Prefecture }\end{array}$ & Plant & Ise Chemical Industries Co. Ltd. & Asahi Glass Co. Ltd., 52.4\%. \\
\hline 939 & Iodine, crude & $\begin{array}{l}\text { Oami-Shirasato, Chiba } \\
\text { Prefecture }\end{array}$ & Plant & Ise Chemical Industries Co. Ltd. & Asahi Glass Co. Ltd., 52.4\%. \\
\hline 941 & Iodine, crude & $\begin{array}{l}\text { Chosei, Chiba } \\
\text { Prefecture }\end{array}$ & Plant & Godo Shigen Sangyo Co. Ltd. & $\begin{array}{l}\text { Kanto Natural Gas } \\
\text { Development Co. Ltd., 11\%. }\end{array}$ \\
\hline 942 & Iodine, crude & Isumi, Chiba Prefecture & Plant & Nippon Chemicals Co. Ltd. & $\begin{array}{l}\text { Nippon Shokubai Co. Ltd., } \\
\text { 17\%. }\end{array}$ \\
\hline 943 & Iodine, crude & $\begin{array}{l}\text { Kurosaki, Niigata } \\
\text { Prefecture }\end{array}$ & Plant & Toho Earthtech, Inc. & Itochi Corp., 34.1\%. \\
\hline
\end{tabular}




\begin{tabular}{|c|c|c|c|c|c|}
\hline $\begin{array}{l}\text { Facility no. } \\
\text { (fig. 1) }\end{array}$ & $\begin{array}{l}\text { Mineral } \\
\text { commodity }\end{array}$ & Location & $\begin{array}{l}\text { Facility } \\
\text { type }\end{array}$ & Operating company & Principal owner \\
\hline 944 & Iodine, crude & $\begin{array}{l}\text { Mobara, Chiba } \\
\text { Prefecture }\end{array}$ & Plant & $\begin{array}{l}\text { Kanto Natural Gas Development } \\
\text { Co. Ltd. }\end{array}$ & Mitsui Chemicals, Inc., 21.9\%. \\
\hline 945 & Iodine, crude & $\begin{array}{l}\text { Shirako, Chiba } \\
\text { Prefecture }\end{array}$ & Plant & Nihon Tennen Gas Co. Ltd. & $\begin{array}{l}\text { Kanto Natural Gas } \\
\text { Development Co. Ltd., 50\%. }\end{array}$ \\
\hline 946 & Iodine, crude & $\begin{array}{l}\text { Yokoshiba, Chiba } \\
\text { Prefecture }\end{array}$ & Plant & Nihon Tennen Gas Co. Ltd. & $\begin{array}{l}\text { Kanto Natural Gas } \\
\text { Development Co. Ltd., 50\%. }\end{array}$ \\
\hline 947 & $\begin{array}{l}\text { Iron and steel, } \\
\text { crude steel }\end{array}$ & $\begin{array}{l}\text { Chiba, Chiba } \\
\text { Prefecture }\end{array}$ & Plant & JFE Steel Corp. & JFE Holdings Inc., 100\%. \\
\hline 950 & $\begin{array}{l}\text { Iron and steel, } \\
\text { crude steel }\end{array}$ & $\begin{array}{l}\text { Kawasaki (Keihin), } \\
\text { Kanagawa } \\
\text { Prefecture }\end{array}$ & Plant & JFE Steel Corp. & JFE Holdings Inc., 100\%. \\
\hline 953 & $\begin{array}{l}\text { Iron and steel, } \\
\text { crude steel }\end{array}$ & $\begin{array}{l}\text { Kimitsu, Chiba } \\
\text { Prefecture }\end{array}$ & Plant & Nippon Steel Corp. & NA. \\
\hline 957 & $\begin{array}{l}\text { Iron and steel, } \\
\text { crude steel }\end{array}$ & $\begin{array}{l}\text { Kashima, Ibaraki } \\
\text { Prefecture }\end{array}$ & Plant & Sumitomo Metal Industries, Ltd. & NA. \\
\hline 966 & Lead, refined & $\begin{array}{l}\text { Kosaka, Akita } \\
\text { Prefecture }\end{array}$ & Plant & $\begin{array}{l}\text { Kosaka Smelting and Refining } \\
\text { Co. Ltd. }\end{array}$ & $\begin{array}{l}\text { Dowa Metals and Mining Co. } \\
\text { Ltd., } 100 \% \text {. }\end{array}$ \\
\hline 967 & Lead, refined & $\begin{array}{l}\text { Hosokura, Miyagi } \\
\text { Prefecture }\end{array}$ & Plant & $\begin{array}{l}\text { Hosokura Smelting and Refining } \\
\text { Mining Co. Ltd. }\end{array}$ & $\begin{array}{l}\text { Mitsubishi Materials Corp., } \\
100 \% \text {. }\end{array}$ \\
\hline 970 & Limestone & $\begin{array}{l}\text { Ofunato, Iwate } \\
\text { Prefecture }\end{array}$ & Mine & Taiheiyo Cement Co. Ltd. & NA. \\
\hline 973 & Limestone & $\begin{array}{l}\text { Taiheiyo Buko, } \\
\text { Saitama Prefecture }\end{array}$ & Mine & Taiheiyo Cement Co. Ltd. & NA. \\
\hline 976 & Limestone & $\begin{array}{l}\text { Hanezuru, Tochigi } \\
\text { Prefecture }\end{array}$ & Mine & Nittetsu Mining Co. Ltd. & NA. \\
\hline 985 & Limestone & $\begin{array}{l}\text { Karasuyama, Tochigi } \\
\text { Prefecture }\end{array}$ & Mine & $\begin{array}{l}\text { Sumitomo-Osaka Cement Co. } \\
\text { Ltd. }\end{array}$ & NA. \\
\hline 1000 & $\begin{array}{l}\text { Titanium } \\
\text { dioxide }\end{array}$ & $\begin{array}{l}\text { Onahama, Fukushima } \\
\text { Prefecture }\end{array}$ & Plant & $\begin{array}{l}\text { Sakai Chemical Industries Co. } \\
\text { Ltd. }\end{array}$ & NA. \\
\hline 1005 & $\begin{array}{l}\text { Titanium, } \\
\text { sponge } \\
\text { metal }\end{array}$ & $\begin{array}{l}\text { Chigasaki, Kanagawa } \\
\text { Prefecture }\end{array}$ & Plant & Toho Titanium Co. Ltd. & $\begin{array}{l}\text { Nippon Mining and Metals Co. } \\
\text { Ltd., } 47 \% \text {. }\end{array}$ \\
\hline 1006 & Zinc, refined & Iijima, Akita Prefecture & Plant & Akita Zinc Co. Ltd. & $\begin{array}{l}\text { Dowa Metals and Mining Co. } \\
\text { Ltd., 81\%. }\end{array}$ \\
\hline 1007 & Zinc, refined & $\begin{array}{l}\text { Annaka, Gunma } \\
\text { Prefecture }\end{array}$ & Plant & Toho Zinc Co. Ltd. & NA. \\
\hline
\end{tabular}




\section{Requirements for Operation of the Mines and Mineral Processing Facilities}

Maintaining or resuming operations at the facilities listed earlier in this report will depend not only on whether the facilities sustained damage from the earthquake and tsunami but also upon the condition of the surrounding infrastructure, including electric power and transportation systems (ports, railroads, and roads), necessary to obtain raw materials and to deliver finished products, as well as on reassembling the workforce that operates the facilities.

Mineral extraction and processing facilities are large consumers of electricity. When operating at full capacity, these facilities consume about 2.5 percent of Japan's production of electricity. A number of the facilities, which were not damaged by the earthquake, have limited activity or ceased operation owing to restrictions on the use of electricity. Figures 2 and 3 show the effect of the earthquake on electrical power availability as indicated by lights observed in images produced by the Earth Observation Group of the National Oceanic and Atmospheric Administration on the nights of March 9 and 12.

Figure 1 shows the location of railroads and ports in the region. Popular media reports indicated damage to the ports at Hitachi, Ishinomaki, Kamaishi, Kashima, Kesennuma, Miyako, Ofunato, Onagawa, Sendai, Soma, and Shiogama. In addition, damage was sustained at the port of Hachinohe, which is north of the area shown on the map in figure 1. 


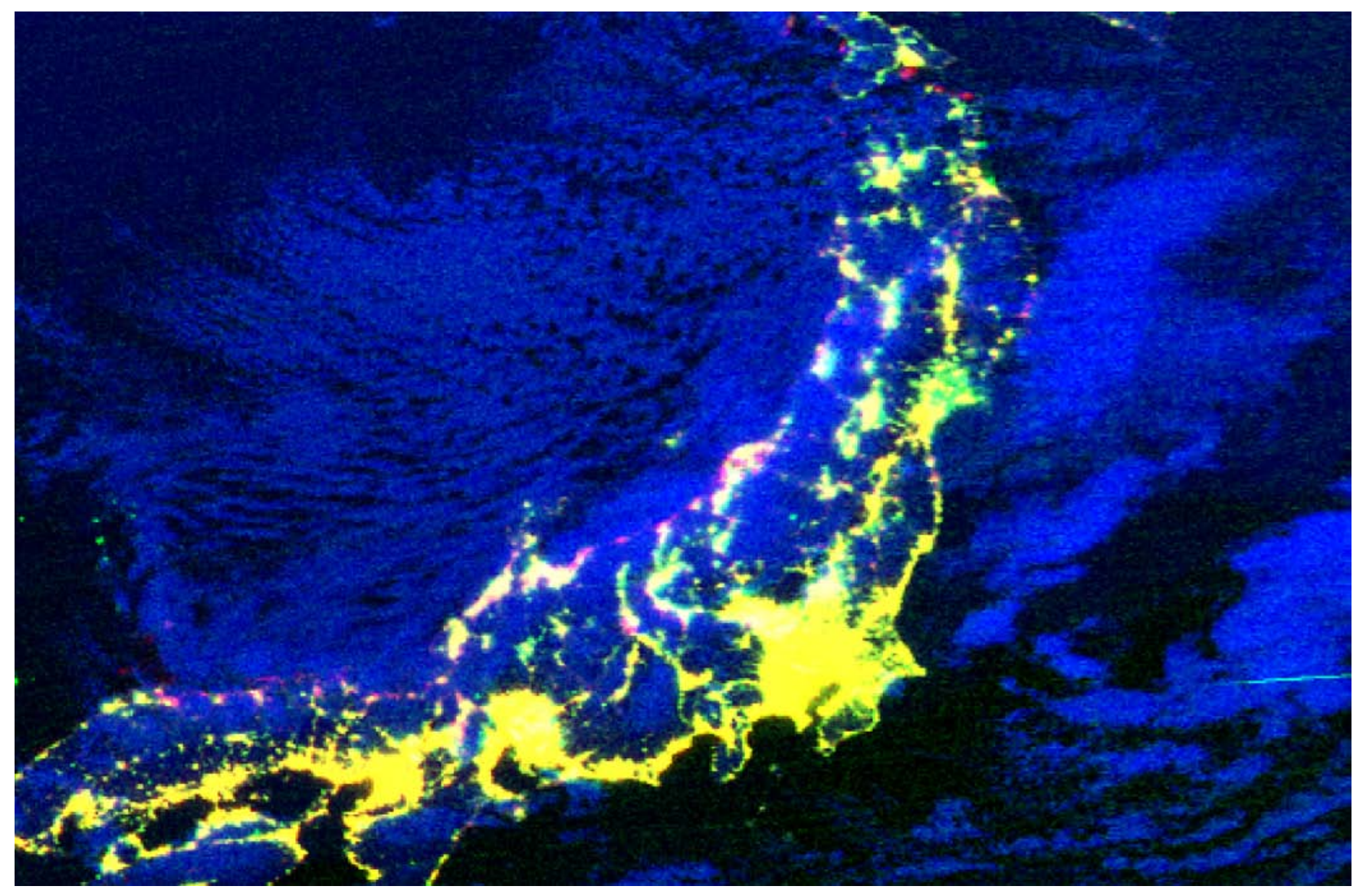

Figure 2. Composite color image by the Earth Observation Group of the National Geophysical Center, National Oceanic and Atmospheric Administration from satellite data captured at 10:07 UTC on March 9, 2011, from U.S. Air Force Defense Meteorological Satellite Program satellite F-18. The yellow color on the map shows lights, magenta indicates lights obscured by clouds, light blue shows clouds, dark blue is the ocean, red indicates locations under a power outage, and green is lighting diffused by cloud cover. 


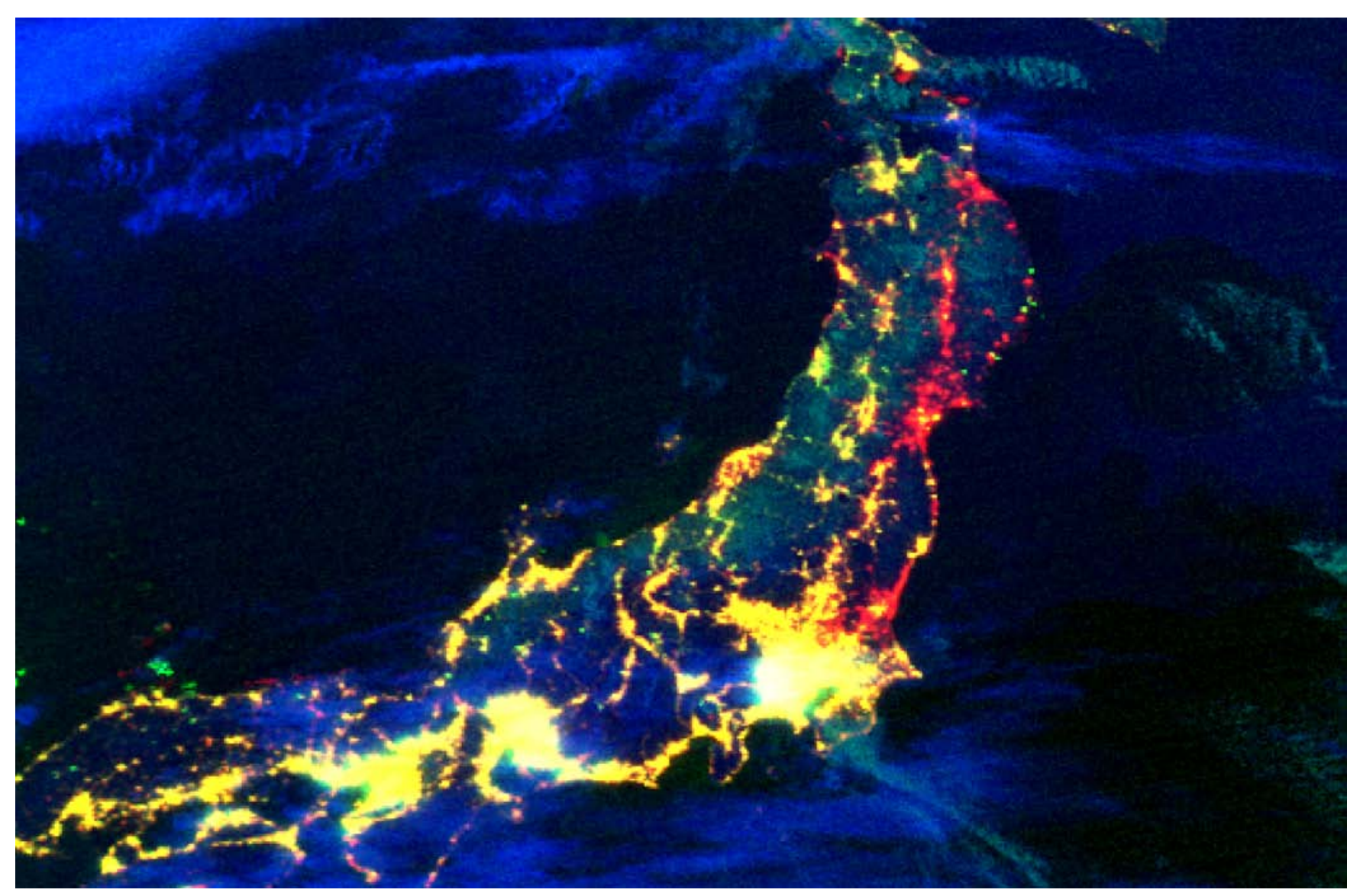

Figure 3. Composite color image by the Earth Observation Group of the National Geophysical Center, National Oceanic and Atmospheric Administration from satellite data captured at 21:30 UTC on March 12, 2011, (one day after the earthquake) from U.S. Air Force Defense Meteorological Satellite Program satellite F-18 The yellow color on the map shows lights, magenta indicates lights obscured by clouds, light blue shows clouds, dark blue is the ocean, red indicates locations under a power outage, and green is lighting diffused by cloud cover.

\section{Conclusions}

Understanding the long term effects of the March 11th earthquake and tsunami is only beginning. According to The Economist (2011), the economic impact of natural disasters is often shortlived; however, "earthquakes have small but consistently negative effects on economic growth" owing to destruction of factories, transportation infrastructure, and electrical systems. In the short-term, lost production of mineral commodities will likely be felt in Japan and beyond.

\section{References Cited}

Baker, M.S., Elias, Nurudeen, Guzmán, Eric, and Soto-Viruet, Yadira, 2010, Mineral facilities of Asia and the Pacific: U.S. Geological Survey Open-File Report 2010-1254, 1 pl., 51-p. table, available only at http://pubs.usgs.gov/of/2010/1254/.

Economist, The, 2011, The cost of calamity: The Economist, March 17, p. 86. (Accessed March 22, 2011, at http://www.economist.com/node/18387016.) 